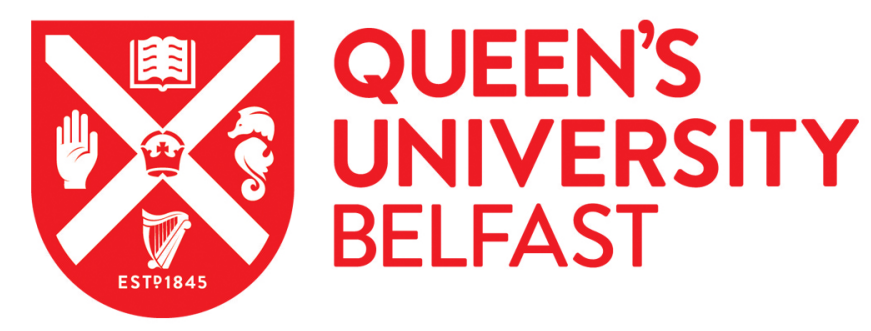

\title{
Experimental Determination of Irreversible Entropy Production in out- of-Equilibrium Mesoscopic Quantum Systems
}

Brunelli, M., Fusco, L., Landig, R., Wieczorek, W., Hoelscher-Obermaier, J., Landi, G., Semiao, F. L., Ferraro, A., Donner, T., Kiesel, N., De Chiara, G., \& Paternostro, M. (2018). Experimental Determination of Irreversible Entropy Production in out-of-Equilibrium Mesoscopic Quantum Systems. Physical Review Letters, 121, [160604]. https://doi.org/10.1103/PhysRevLett.121.160604

Published in:

Physical Review Letters

Document Version:

Peer reviewed version

Queen's University Belfast - Research Portal:

Link to publication record in Queen's University Belfast Research Portal

Publisher rights

(C) 2018 APS.

This work is made available online in accordance with the publisher's policies. Please refer to any applicable terms of use of the publisher.

\section{General rights}

Copyright for the publications made accessible via the Queen's University Belfast Research Portal is retained by the author(s) and / or other copyright owners and it is a condition of accessing these publications that users recognise and abide by the legal requirements associated with these rights.

Take down policy

The Research Portal is Queen's institutional repository that provides access to Queen's research output. Every effort has been made to ensure that content in the Research Portal does not infringe any person's rights, or applicable UK laws. If you discover content in the Research Portal that you believe breaches copyright or violates any law, please contact openaccess@qub.ac.uk. 


\title{
Experimental determination of irreversible entropy production in out-of-equilibrium mesoscopic quantum systems
}

\author{
M. Brunelli, ${ }^{1}$ L. Fusco, ${ }^{2}$ R. Landig, ${ }^{3,}{ }^{*}$ W. Wieczorek,${ }^{4}$ J. Hoelscher-Obermaier, ${ }^{5,6}$ G. Landi, ${ }^{7}$ \\ F. L. Semião, ${ }^{8}$ A. Ferraro, ${ }^{2}$ N. Kiesel, ${ }^{5}$ T. Donner, ${ }^{3}$ G. De Chiara, ${ }^{2}$ and M. Paternostro ${ }^{2}$ \\ ${ }^{1}$ Cavendish Laboratory, University of Cambridge, Cambridge CB3 OHE, United Kingdom \\ ${ }^{2}$ Centre for Theoretical Atomic, Molecular and Optical Physics, \\ School of Mathematics and Physics, Queen's University, Belfast BT7 1NN, United Kingdom \\ ${ }^{3}$ Institute for Quantum Electronics, ETH Zürich, 8093 Zürich, Switzerland \\ ${ }^{4}$ Department of Microtechnology and Nanoscience, Chalmers University of Technology, 41296 Göteborg, Sweden \\ ${ }^{5}$ University of Vienna, Faculty of Physics, Vienna Center for Quantum Science and Technology (VCQ), Boltzmanngasse 5, 1090 Vienna, Austria \\ ${ }^{6}$ Leibniz University Hannover, Institute for Gravitational Physics (Albert-Einstein-Institute), Callinstraße 38, 30167 Hannover, Germany \\ ${ }^{7}$ Instituto de Física da Universidade de São Paulo, 05314-970 São Paulo, Brazil \\ ${ }^{8}$ Centro de Ciências Naturais e Humanas, Universidade Federal do ABC, 09210-170, Santo André, São Paulo, Brazil
}

\begin{abstract}
By making use of a recently proposed framework for the inference of thermodynamic irreversibility in bosonic quantum systems, we experimentally measure and characterize the entropy production rates in the nonequilibrium steady state of two different physical systems - a micro-mechanical resonator and a Bose-Einstein condensate - each coupled to a high finesse cavity and hence also subject to optical loss. Key features of our setups, such as cooling of the mechanical resonator and signatures of a structural quantum phase transition in the condensate are reflected in the entropy production rates. Our work demonstrates the possibility to explore irreversibility in driven mesoscopic quantum systems and paves the way to a systematic experimental assessment of entropy production beyond the microscopic limit.
\end{abstract}

Entropy is a crucial quantity for the characterisation of dynamical processes: it quantifies and links seemingly distant notions such as disorder, information, and irreversibility across different disciplinary boundaries [1,2]. Every finitetime transformation results in some production of entropy, which signals the occurrence of irreversibility. Quantifying the amount of irreversible entropy produced by a given process is a goal of paramount importance: entropy production is a key quantity for the characterisation of non-equilibrium processes, and its minimisation improves the efficiency of thermal machines. The second law of thermodynamics can be formulated in terms of a universal constraint on the entropy production, which can never be negative $[3,4]$. In turn, this leads to the following rate equation for the variation of the entropy $S$ [5]

$$
\frac{d S}{d t}=\Pi(t)-\Phi(t)
$$

where $\Pi(t)$ and $\Phi(t)$ are the irreversible entropy production rate and the entropy flux from the system to the environment, respectively. When the system reaches a non-equilibrium steady-state (NESS) these quantities take values $\Pi_{s}$ and $\Phi_{s}$ respectively, such that $\Pi_{s}=\Phi_{s}>0$ [see Fig. 1 (a)]. Under these conditions, entropy is produced and exchanged with the local baths at the same rate. Only when both terms vanish $\left(\Pi_{s}=\Phi_{s}=0\right)$ one recovers thermal equilibrium. The entropy production rate directly accounts for the irreversibility of a process and uncovers the non-equilibrium features of a system.

The link between the entropy production rate $\Pi_{s}$ and irreversibility becomes particularly relevant in small systems subjected to fluctuations, for which a microscopic definition of entropy production based on stochastic trajectories of the system has been given [6]. Experimentally, this notion has been used to test fluctuation theorems in a variety of classically operating systems such as a single-electron box [7], a two-level system driven by a time-dependent potential [8], and a levitated nanoparticle undergoing relaxation [9]. However, in order to harness the working principles of thermodynamic machines working at the quantum level, and pinpoint the differences between their performances and those of their classical counterparts, it is important to analyse the entropy generated through genuine quantum dynamics [10]. Moreover, while so far nanoscale systems have been used for the experimental study of classical out-of-equilibrium thermodynamics, irreversible entropy production arising from quantum dynamics in mesoscopic quantum systems has not been experimentally investigated yet.

Recently, progress towards the theoretical characterisation of entropy production in bosonic systems brought out of equilibrium has been made [11-13]. In this paper, we make use of such theoretical framework to quantify experimentally the amount of irreversibility in the NESS of two different drivendissipative quantum systems, realized by coupling bosonic systems to high-finesse cavities. The light field mode of a cavity allows to infer the entropy production in terms of relevant controllable parameters of the coupled system. In particular, in this study, we investigate the influence of different dynamical regimes and sources of environmental noise on the quantum fluctuations of a quantum system, and thus the corresponding entropy production rate. In order to address such influences, we assess two distinct experimental setups: a cavity-optomechanical (cavity-OM) device and a Bose-Einstein condensate (BEC) with cavity-mediated longrange interactions [14-16]. The required measurements are based on the spectra of the light fields leaking out of the respective cavities. Remarkably, the entropy production reflects 
(a)
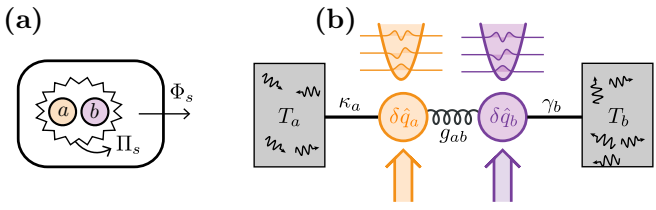

(c)

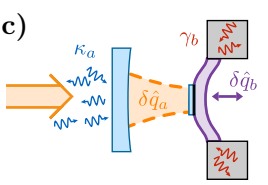

(d)

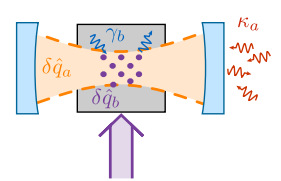

FIG. 1. (a) The driven-dissipative system, consisting of the coupled subsystems $a$ and $b$, reaches a NESS with an associated entropy production rate $\Pi_{s}$ and an entropy flux $\Phi_{s}$ from the system to the environment. (b) Both systems can be modelled as two quantum harmonic oscillators at frequencies $\omega_{a}$ and $\omega_{b}$, linearly coupled with a strength $g_{a b}$. Each oscillator is coupled to independent local baths at temperature $T_{a}$ and $T_{b}$, respectively. The corresponding coupling rates are $\kappa_{a}$ and $\gamma_{b}$. The oscillators can be pumped by an external field (purple and orange arrows in the figure). (c) Optomechanical setup: a micro-mechanical oscillator $\left(\delta \hat{q}_{b}\right)$ is coupled to the field mode of an optical Fabry-Perot cavity $\left(\delta \hat{q}_{a}\right)$. For this setup only the cavity is pumped. (d) Cavity-BEC setup: the external degree of freedom of a BEC $\left(\delta \hat{q}_{b}\right)$ is coupled to the field mode of a cavity $\left(\delta \hat{q}_{a}\right)$. For this setup only the atoms are pumped. Red and blue wiggly lines indicate heating or cooling of the subsystems via coupling to the baths. In both setups the number of excitations in the optical bath is zero, i.e. $n_{T_{a}}=0$.

the specific features of the two experimental platforms, which are very different in nature despite the common description provided here. As such, our results show how a key indicator of irreversibility is fully within the grasp of dynamically controlled quantum dynamics.

In cavity-OM systems, the cavity photon number is coupled to the position of the mechanical oscillator [cf. Fig. 1(b) and (c)]. Our specific implementation uses a Fabry-Perot cavity. One of its mirrors is a doubly clamped, highly reflective, mechanical cantilever. Radiation pressure couples the intracavity photon number to the position of the cantilever. The mechanical support of the cantilever provides a local heat bath at room temperature. The optical cavity is driven by a laser that is red-detuned by the mechanical frequency from the optical cavity resonance. For a driving laser without classical noise, the cavity mode is coupled to a zero-excitation heat bath. We observe sideband cooling of the mechanical motion [17-20] and, for large drive powers, strong optomechanical coupling [21-23]. To analyse the entropy production rate of the cavity-OM system, we measure the light reflected off the cavity via homodyne detection.

Also in the second implementation, the two coupled harmonic oscillators correspond to a light field mode coupled to a mechanical degree of freedom [cf. Fig 1(b) and (d)]. We load a BEC into a high-finesse optical cavity and illuminate the atoms with a standing-wave transverse laser field. Far-off resonant scattering of photons from the laser field into a near-

\begin{tabular}{|c|c|c|c|c|c|c|}
\hline & $\begin{array}{c}\omega_{a} / 2 \pi \\
{[\mathrm{MHz}]}\end{array}$ & $\begin{array}{l}\kappa_{a} / 2 \pi \\
{[\mathrm{kHz}]}\end{array}$ & $\begin{array}{c}\omega_{b} / 2 \pi \\
{[\mathrm{kHz}]}\end{array}$ & $\begin{array}{c}\gamma_{b} / 2 \pi \\
{[\mathrm{Hz}]}\end{array}$ & $\begin{array}{c}T_{b} \\
{[\mathrm{~K}]}\end{array}$ & $\begin{array}{c}\text { Other } \\
\text { parameters }\end{array}$ \\
\hline cavity-OM & 1.27815 & 435.849 & 1278.15 & 264.1 & 292 & $m=176 \mathrm{ng}$ \\
\hline cavity-BEC & 15.13 & 1250 & 8.3 & [25] & $38 \times 10^{-9}$ & $N=10^{5}$ \\
\hline
\end{tabular}

TABLE I. Physical parameters for the two experimental setups. The damping rate $\gamma_{b}$ is constant in the cavity-OM experiment, while in the cavity-BEC setup it depends on the actual working point (cf. Ref. [25] for details). Here, $m$ is the effective mass of the mechanical oscillator, and $N$ is the number of ${ }^{87} \mathrm{Rb}$ atoms in the BEC.

detuned, initially empty cavity field mode, couples the zeromomentum mode of the BEC to an excited momentum mode. The scattering process mediates effective atom-atom interactions, which are of long-range, since the photons are delocalized in the cavity mode [16]. This interaction is tunable in strength via the power of the transverse laser beam. The longrange interaction can be brought to competition with the kinetic energy of the atoms, resulting in a structural phase transition [24]. In the spatially homogeneous phase, for increasing interaction, the energy of the excited momentum mode softens, until at a critical interaction strength the system breaks a discrete symmetry and the atoms arrange in a spatially modulated density distribution. The equivalence of this system to a Dicke model has been demonstrated in Ref. [15]. We measure the cavity light field leaking through the mirrors with a heterodyne detection setup. The spectral analysis of this signal is used to infer the diverging amount of atomic density fluctuations accompanying the structural phase transition [24].

In both cases, the effective interaction between the oscillators is obtained by a harmonic expansion of the field operators around their mean values, resulting in two linearly coupled quantum oscillators [cf. Fig. 1(b)]. We denote with $\delta \hat{q}_{a, b}$ and $\delta \hat{p}_{a, b}$ the position and momentum fluctuation operators around the mean-field values of the two oscillators. In what follows, $a$ and $b$ refer to the optical and mechanical/atomic os-
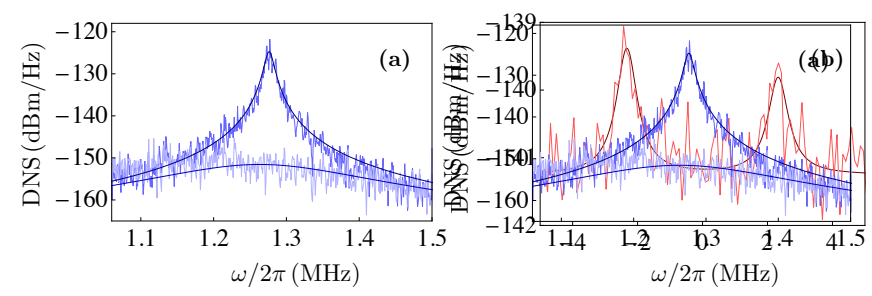

FIG. 2. Experimental density noise spectra. Panel (a): Density noise spectrum (DNS) of the phase quadrature of the output cavity field, attenuated before detection, for the cavity-OM setup. The jagged blue curve refers to a value of the rescaled coupling $g_{a b} / \kappa_{a}=0.49$, while the jagged light-blue curve to $g_{a b} / \kappa_{a}=2.29$. The fits of the DNS are shown as smooth lines. Notice that the power spectrum is originally dimensionless, and has been here converted to SI units for uniformity of notation. Panel (b): DNS of the extra-cavity field for the cavity-BEC system at a coupling $\left(g_{a b} / g_{a b}^{\text {cr }}\right)^{2}=0.93$. A fit of the DNS is shown as a smooth line. 
cillators, respectively. In a frame rotating at the frequency $\omega_{p}$ of the respective pump fields, the oscillators have frequencies $\omega_{a}=\omega_{c}-\omega_{p}$ and $\omega_{b}$ (here $\omega_{c}$ is the frequency of the cavity field). Their interaction is described by the Hamiltonian

$$
\hat{H}=\frac{\hbar \omega_{a}}{2}\left(\delta \hat{q}_{a}^{2}+\delta \hat{p}_{a}^{2}\right)+\frac{\hbar \omega_{b}}{2}\left(\delta \hat{q}_{b}^{2}+\delta \hat{p}_{b}^{2}\right)+\hbar g_{a b} \delta \hat{q}_{a} \delta \hat{q}_{b},
$$

where $g_{a b}$ is the coupling strength between the modes. In the superradiant phase of the Dicke model, an additional squeezing term of the atomic mode must be included in the Hamiltonian [16]. For the derivation of the models and the values of the parameters in the two setups, we refer to Refs. [25] and to Table I. The systems are inherently open: each harmonic oscillator is independently coupled to a local bath. This provides both a dissipation channel and extra quantum fluctuations in addition to those present in the closed systems. The optical cavity mode is coupled to the surrounding electromagnetic vacuum with a decay rate $\kappa_{a}$. On the other hand, the nature of the mechanical/atomic bath is specific to the setup being considered. In the cavity-OM system, the coupling of the vibrating mirror to the background of phonon modes is described in terms of quantum Brownian motion. In the cavityBEC system, dissipation is due to the collection of excited Bogolioubov modes, which provides a bath for the condensate. In both cases, we assume oscillator $b$ to be in contact with a Markovian bath at temperature $T_{b}$ and rate $\gamma_{b}$. The average number of excitations in the equilibrium state of oscillator $b$ is thus $n_{T_{b}}=\left(e^{\hbar \omega_{b} / k_{B} T_{b}}-1\right)^{-1}$ (cf. Ref [34]). The driven-dissipative nature of the systems is such that a NESS is eventually reached $[14,26]$.

The linear dynamics undergone by the coupled oscillators allows us to exploit a framework developed for linear stochastic processes [11-13]. In particular, the situation that we face is perfectly suited to the use of the framework for the quantification of entropy production proposed in Ref. [12], where the entropy $S$ of an arbitrary bosonic quantum system prepared in a Gaussian state is written in terms of the Shannon entropy of the Wigner function

$$
S(t)=-\int \mathcal{W}(u, t) \log \mathcal{W}(u, t) d u
$$

where $\mathcal{W}(u, t)$ is the Wigner function at time $t$ corresponding to the state of the two oscillators, and $u$ is the corresponding vector of complex phase-space variables. The quadratic nature of Eq. (2) and the initial thermal state of the oscillators in both setups ensures the positivity of $\mathcal{W}(u, t)$ and allows us to write it in terms of the variances of the fluctuation operators of the oscillators, which enormously simplifies the explicit calculation of $\Pi(t)$. In the NESS, all entropy produced in the system flows to the environments so that $\Pi_{s}=\Phi_{s}$. Following the lines sketched in Ref. [25], the entropy production rate in the NESS due to the quantum fluctuations takes the form

$$
\Pi_{s}=\Phi_{s}=2 \gamma_{b}\left(\frac{n_{b}+1 / 2}{n_{T_{b}}+1 / 2}-1\right)+4 \kappa_{a} n_{a}=\mu_{b}+\mu_{a},
$$

where $n_{a}=\left\langle\left(\delta \hat{q}_{a}^{2}+\delta \hat{p}_{a}^{2}-1\right)\right\rangle_{s} / 2$ and $n_{b}=\left\langle\left(\delta \hat{q}_{b}^{2}+\delta \hat{p}_{b}^{2}-1\right)\right\rangle_{s} / 2$ are the average number of excitations in the NESS of the two oscillators in excess of the zero-point motion of the respective harmonic oscillator. In the cavity-OM expression for $\mu_{b}$, instead of the full phonon number $n_{b}$, only the momentum variance $\left\langle\delta \hat{p}_{b}^{2}\right\rangle_{s}$ enters as we assume Brownian motion damping.

Eq. (4) represents our main theoretical result: it quantifies the entropic contribution, ascribable to the quantum fluctuations that the system has to pay to remain in its NESS. It thus directly quantifies the irreversibility of the driven-dissipative dynamics of two linearly coupled quantum oscillators, well beyond the linear-response limit. For vanishing coupling the systems reach thermal equilibrium (i.e. $n_{a}=0$ and $n_{b}=n_{T_{b}}$ ), and $\Pi_{s}$ vanishes. Moreover, there is no dependence on the correlations between the oscillators, since in a NESS the entropy production rate $\Pi_{s}$ equals the flux rate $\Phi_{s}$. Thus, the entropy flux from the system to the overall environment determines the amount of irreversibility produced within the driven-dissipative model, and is directly linked to the breaking down of the microscopic detailed balance [10]. The previous considerations also allow us to identify two contributions to $\Pi_{s}$, linked to the mechanical/atomic and optical oscillator, referred to as $\mu_{a}$ and $\mu_{b}$, respectively. They are the individual entropy flows to each environment and show how the entropy produced in the NESS is split into two distinct fluxes. We note that the explicit form of Eq. (4) in terms of the sum of such independent terms strongly relies on the local nature of the environments that we have cconsidered, and we expect it not to hold in more general situations. The dissipative evolution arising from the contact with the environments is manifested explicitly in Eq. (4) by the presence of the rates $\gamma_{b}$ and $\kappa_{a}$. In both settings, the mechanical/atomic damping rate $\gamma_{b}$ is much smaller than the cavity decay rate $\kappa_{a}$, as can be appreciated from Table I.

A general formulation of entropy production demands the knowledge of the global state of the system [27-31]. However, $\Pi_{s}$ evaluated for the linearised dynamics in Eq. (2) only involves the mean excitations of the oscillators $[11,13]$. For the experimental regime of interest, the dynamics of the cavity field adiabatically follows the mechanical/atomic mode. By measuring the light field leaking out of the cavity we thus can infer about both $\mu_{a}$ and $\mu_{b}$. For both experimental setups, the coupling $g_{a b}$ is varied by increasing the power of the pump. The density noise spectrum (DNS) of the cavity field quadratures is recorded $[24,32]$. Typical examples of the experimental DNS, together with the fitting curves used for their analysis, are shown in Fig. 2. In the cavity-OM experiment, the datasets are taken for $\omega_{a}=\omega_{b}$, which is the working point where the cooling of the mechanical resonator is most effective in the resolved-sideband regime. In the cavity-BEC experiment, on the other hand, the parameters are $\omega_{a} \gg \omega_{b}$, resulting in only a tiny admixture of the optical subsystem. A further difference between the two platforms is in the way the two oscillators are populated: in the optomechanical case, we have $n_{b} \gg n_{a}$ for the lowest coupling values, while they become comparable in size for the maximum cooling achieved. 

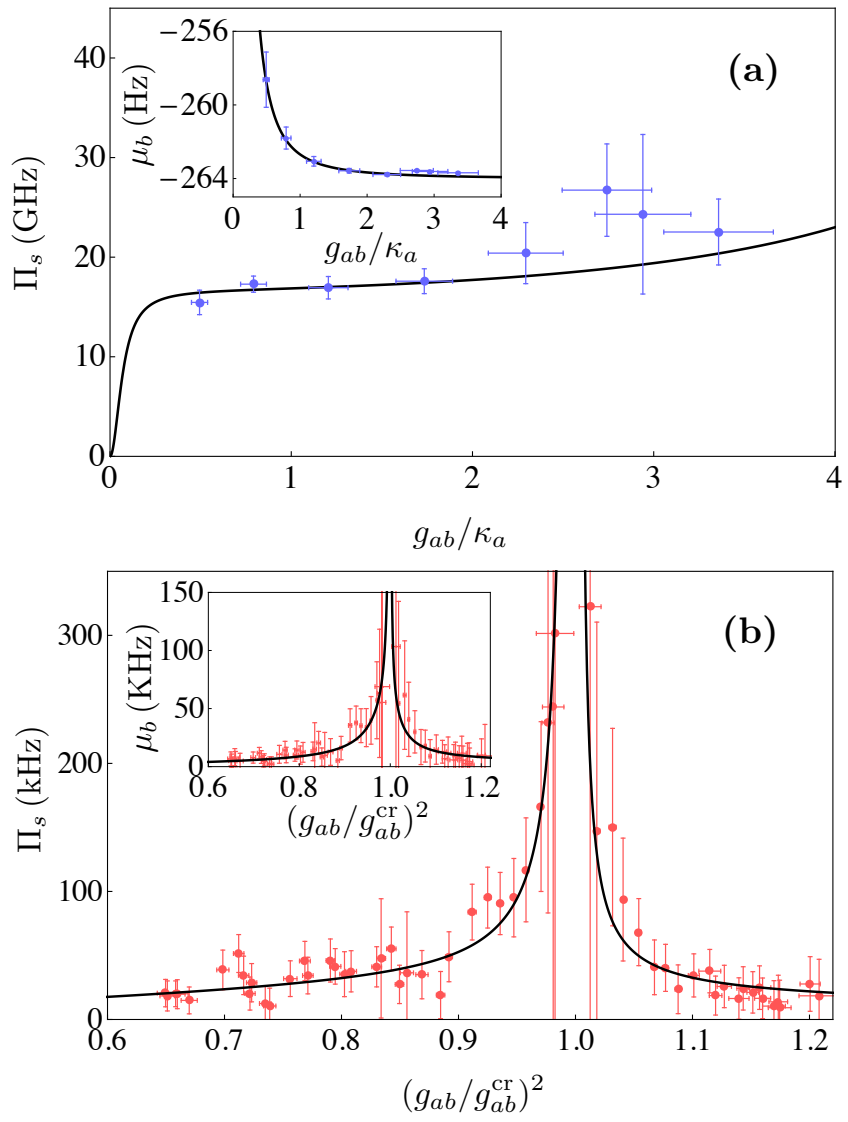

FIG. 3. Experimental assessment of the irreversible entropy production rate $\Pi_{s}$ at the NESS for (a) the cavity-OM system and (b) the cavity BEC system. In the cavity-OM system, $g_{a b}$ is twice the standard optomechanical coupling rate $[14,25]$. For the cavity-BEC setup, the control parameter $g_{a b}$ is renormalised with respect to the critical parameter $g_{a b}^{\text {cr }}=\sqrt{\left(\kappa_{a}^{2}+\omega_{a}^{2}\right) \omega_{b} / 4 \omega_{a}}$. The insets show the behaviour of $\mu_{b}$ in each of the settings considered. In both panels, the solid black lines show the theoretical predictions based on the values given in Table I. The blue and red dots show the experimental data for the cavity-OM and cavity-BEC experiment, respectively. In panel (a), the vertical error bars report statistical errors extracted from the fit, while the horizontal ones show experimental error on the values of the parameter. In panel (b), the vertical and horizontal error bars report the statistical errors from the fit and the determination of the critical point, respectively [24].

In the cavity-BEC setup, the cavity field is considerably less populated than the atomic mode. Finally, the mechanical bath is at room temperature, while the temperature of the atomic reservoir is below the condensation point and in the $\mathrm{nK}$ range (cf. Table I). This highlights and reinforces the diversity of the experimental platforms that we have addressed within a unique framework for the quantification of irreversible entropy.

Following the technical approach illustrated in Refs. [1113] and sketched in [25], we have separately reconstructed the two terms $\mu_{a}$ and $\mu_{b}$ that determine quantitatively $\Pi_{s}$. Fig. 3 displays the experimental data together with the theoretical model, demonstrating a very good quantitative agree- ment. Besides the influences of the environments, the entropy production rates depends on the interplay between the mutual dynamics of the oscillators. For the cavity-OM system, the contribution to $\Pi_{s}$ we observe from the mechanical oscillator is much smaller than the one coming from the optical field. On the contrary, $\mu_{a} \simeq \mu_{b}$ in the atomic setup. For each of the two experiments $\Pi_{s}$ is positive, in agreement with the second law. In the cavity-OM setup, $\mu_{a}$ is an increasing function of the coupling: the stronger the pump, the further the system operates away from thermal equilibrium and the more entropy is generated. At the same time, $\mu_{b}$ takes negative values, whose magnitude increases for increasing values of $g_{a b}$. This is legitimate as $\mu_{b}$ is not per se an entropy production rate but represents an individual flux, which can thus take negative values (while $\mu_{a}+\mu_{b}$ has to be positive). The observed behaviour of $\mu_{b}$ is a signature of optomechanical cooling: its growth, in absolute value, with $g_{a b}$ shows the increase of the entropy flow from the mechanical resonator to the cavity field, corresponding to lowering of the effective temperature of the resonator. As for the cavity-BEC system, the divergent behaviour of the entropy production rate at the critical point reflects the occurrence of the structural phase transition: at $g_{a b}^{\mathrm{cr}}$, the known divergence of the populations of the two oscillators at the steady-state [33] results in the singularity of both $\mu_{a}$ and $\mu_{b}$ separately. The irreversible entropy production rate thus diverges at criticality.

We have experimentally determined the entropy production rate, a key indicator of irreversibility, in driven-dissipative quantum systems operating at the steady-state. The two experimental setups, being instances of mesoscopic systems undergoing quantum dynamics, allowed us to link the phenomenology of the entropy production rate to the salient features of their physics. We have thus assessed architectures that could embody the building blocks of a generation of future thermodynamic machines working out of equilibrium, and thus subjected to irreversible processes. For such devices, the quantification of irreversibility will be very relevant for the characterisation of their efficiency, as it will provide useful information to design protocols able to quench it, thus optimising their working principles.

Acknowledgements.- We are grateful to M. Aspelmeyer, T. Esslinger, J. Goold, I. Lesanovsky, E. Lutz, and J. Schmiedmayer for useful comments and fruitful discussions during the development of this project. We thank S. Gröblacher for support with microfabrication, and F. Brennecke and R. Mottl for support in taking and evaluating the cavity-BEC data. This work was supported by the European Union through the projects TherMiQ, TEQ, SIQS, iQOEMS, and ITN cQOM, the European Research Council through the Advanced Grant project SQMBS, the Brazilian CNPq through Grant number 302900/2017-9 and the "Ciência sem Fronteiras" programme via the "Pesquisador Visitante Especial" initiative (Grant number 401265/2012-9), the São Paulo Research Foundation (FAPESP) under grant number 2014/01218-2, the Brazilian National Institute of Science and Technology of Quantum Information (INCT/IQ), the Vienna Science and Technology 
Fund (WWTF, Project No. ICT12-049), and the Austrian Science Fund FWF (JHO: W1210, CoQuS; NK: AY0095221, START).

* Present address: Department of Physics, Harvard University, Cambridge, Massachusetts 02138, USA.

[1] J. P. Sethna, Entropy, Order Parameters, and Complexity, Oxford University Press (2011).

[2] M. W. Zemansky, Heat and Thermodynamics, McGraw-Hill Book Company Inc. (1968).

[3] U. Seifert, Stochastic thermodynamics fluctuation theorems and molecular machines, Rep. Prog. Phys. 75, 126001 (2012).

[4] C. Jarzynski, Equalities and Inequalities: Irreversibility and the Second Law of Thermodynamics at the Nanoscale, Annu. Rev. Condens. Matter Phys. 2, 329 (2011).

[5] R. C. Tolman and P. C. Fine, On the Irreversible Production of Entropy, Rev. Mod. Phys. 20, 51 (1948).

[6] U. Seifert, Entropy Production along a Stochastic Trajectory and an Integral Fluctuation Theorem, Phys. Rev. Lett. 95, 040602 (2005).

[7] J. V. Koski, T. Sagawa, O-P. Saira, Y. Yoon, A. Kutvonen, P. Solinas, M. Möttönen, T. Ala-Nissila and J. P. Pekola, Distribution of entropy production in a single electron box, Nature Phys. 9, 644-648 (2013).

[8] C. Tietz, S. Schuler, T. Speck, U. Seifert, and J. Wrachtrup, Measurement of Stochastic Entropy Production, Phys. Rev. Lett. 97, 050602 (2006).

[9] J. Gieseler, R. Quidant, C. Dellago and L. Novotny, Dynamic relaxation of a levitated nanoparticle from a non-equilibrium steady state, Nature Nanotech. 9, 358 (2014).

[10] T. B. Batalhão, A. M. Souza, R. S. Sarthour, I. S. Oliveira, M. Paternostro, E. Lutz, and R. M. Serra, Phys. Rev. Lett. 115, 190601 (2015).

[11] G. T. Landi, T. Tomé, and M. J. de Oliveira, Entropy production in linear Langevin systems, J. Phys. A: Math. Theor. 46, 395001 (2013).

[12] J. P. Santos, G. Landi, and M. Paternostro, The Wigner entropy production rate, Phys. Rev. Lett. 118, 220601 (2017).

[13] M. Brunelli, and M. Paternostro, arXiv:1610.01172 (2016).

[14] M. Aspelmeyer, T. Kippenberg, and F. Marquardt, Cavity optomechanics, Rev. Mod. Phys. 86, 1391 (2014).

[15] K. Baumann, C. Guerlin, F. Brennecke, T. Esslinger, Dicke quantum phase transition with a superfluid gas in an optical cavity, Nature 464, 1301 (2010).

[16] R. Mottl, F. Brennecke, K. Baumann, R. Landig, T. Donner, and T. Esslinger, Roton-Type Mode Softening in a Quantum Gas with Cavity-Mediated Long-Range Interactions, Science 336, 1570 (2012).

[17] A. Schliesser, R. Rivieère, G. Anetsberger, O. Arcizet, and T. Kippenberg, Resolved-sideband cooling of a micromechanical oscillator, Nature Phys. 4, 415 (2007).

[18] S. Gröblacher, J. B. Hertzberg, M. R. Vanner, G. D. Cole, S. Gigan, K. C. Schwab, and M. Aspelmeyer, Demonstration of an ultracold micro-optomechanical oscillator in a cryogenic cavity, Nature Phys. 5, 485 (2009).

[19] J. Chan, T. P. Mayer Alegre, A. H. Safavi-Naeini, J. T. Hill, A.
Krause, S. Gröblacher, M. Aspelmeyer, and O. Painter, Laser cooling of a nanomechanical oscillator into its quantum ground state, Nature 478, 89 (2011).

[20] J. D. Teufel, T. Donner, Dale Li, J. W. Harlow, M. S. Allman, K. Cicak, A. J. Sirois, J. D. Whittaker, K. W. Lehnert and R. W. Simmonds, Sideband cooling of micromechanical motion to the quantum ground state, Nature 475, 359 (2011).

[21] S. Gröblacher, K. Hammerer, M. R. Vanner and M. Aspelmeyer, Observation of strong coupling between a micromechanical resonator and an optical cavity field, Nature 460, 724 (2009).

[22] E. Verhagen, S. Deléglise, S. Weis, A. Schliesser and T. J. Kippenberg, Quantum-coherent coupling of a mechanical oscillator to an optical cavity mode, Nature 482, 63 (2012).

[23] J. D. Teufel, Dale Li, M. S. Allman, K. Cicak, A. J. Sirois, J. D. Whittaker, and R. W. Simmonds, Circuit cavity electromechanics in the strong-coupling regime, Nature 471, 204 (2011).

[24] R. Landig, F. Brennecke, R. Mottl, T. Donner, and T. Esslinger, Measuring the dynamic structure factor of a quantum gas undergoing a structural phase transition, Nature Commun. 6, 7046 (2015).

[25] See Supplementary Information accompanying this manuscript, and available from XXXX, where additional details on the formal derivation of the rate of entropy production and experimental methods are illustrated, and which includes Refs. [35-39].

[26] F. Brennecke, R. Mottl, K. Baumann, R. Landig, T. Donner, and T. Esslinger, Real-time observation of fluctuations at the driven-dissipative Dicke phase transition, PNAS 110, (29)11763, (2013).

[27] R. E. Spinney, I. J. Ford, Entropy production in full phase space for continuous stochastic dynamics, Phys. Rev. E 85051113 (2012).

[28] G. Crooks, Path-ensemble averages in systems driven far from equilibrium, Phys. Rev. E 612361 (2000).

[29] I. J. Ford and R. E. Spinney, Entropy production from stochastic dynamics in discrete full phase space, Phys. Rev. E 86021127 (2012).

[30] T. Tomé and M. J. de Oliveira, Entropy Production in Nonequilibrium Systems at Stationary States, Phys. Rev. Lett. 108 020601 (2012).

[31] S. Deffner, Quantum entropy production in phase space, EPL 103, 30001 (2013).

[32] W. Wieczorek, S. G. Hofer, J. Hoelscher-Obermaier, R. Riedinger, K. Hammerer, and M. Aspelmeyer, Optimal State Estimation for Cavity Optomechanical Systems, Phys. Rev. Lett. 114, 223601 (2015).

[33] D. Nagy, G. Szirmai, P. Domokos, Critical exponent of a quantum-noise-driven phase transition: The open-system Dicke model, Phys. Rev. A 84, 043637 (2011).

[34] The observed atomic damping, for the cavity-BEC setup, takes place on time scales longer than the time scale of the appearance of polariton modes. Thus the Bose distribution function, for the thermal number of excitation, is evaluated at the softening frequency of the atomic polariton mode (see SI).

[35] G. Adesso, D. Girolami, and A. Serafini, Phys. Rev. Lett. 109, 190502 (2012).

[36] V. Giovannetti, and D. Vitali, Phys. Rev. A 63023812 (2001).

[37] A. A. Clerk, M. H. Devoret, S. M. Girvin, F. Marquardt, and R. J. Schoelkopf, Rev. Mod. Phys. 82, 1155 (2010).

[38] R. H. Dicke, Phys. Rev. 93, 99 (1954).

[39] W. Kopylov, C. Emary, and T. Brandes, Phys. Rev. A 87, 043840 (2013). 